EXTENDED REPORT

\title{
Improved visualisation of choroidal neovascularisation by scanning laser ophthalmoscope using image averaging
}

\author{
D U Bartsch, M H El-Bradey, A El-Musharaf, W R Freeman
}

Br J Ophthalmol 2005;89:1026-1030. doi: 10.1136/bjo.2004.057364

See end of article for authors' affiliations

Correspondence to:

D U Bartsch, PhD, UCSD

Jacobs Retina Center,

Department of

Ophthalmology, University

of California San Diego,

9415 Campus Point Drive, La Jolla, CA 92093-0946,

USA; dbartsch@ucsd.edu

Accepted for publication

1 December 2004

\begin{abstract}
Aims: To improve visualisation of angiographic features in patients with age related macular degeneration associated with choroidal neovascularisation (CNV) and related complications. To evaluate if image averaging can achieve this goal.

Methods: 27 eyes of 20 sequential patients with age related macular degeneration over a 3 month period were studied. Indocyanine green angiograms (ICGA), fluorescein angiograms (FA), and oral fluorescein angiograms were recorded with a confocal scanning laser ophthalmoscope. Software was used to average multiple images from a 10-20 image series (over $0.5-1.0$ seconds). Image quality was assessed by two masked observers and graded on a scale of $0-3$. A more quantifiable grading method was devised by adding a variable amount of Gaussian noise to the improved image until the original and image averaged image appeared equal.

Results: Masked review showed mild to strong improvement of visualisation of structures including borders of CNV. Improvement varied depending on the type and phase of the angiogram. Improvement was highest in late phase FA, mid and late phase ICGA, and all phases of oral FA.

Conclusion: Image averaging using soffware based algorithms improves the quality of angiographic images, particularly late ICGA images and oral FAs. This method may assist in the visualisation of choroidal neovascularisation in age related macular degeneration.
\end{abstract}

$\mathrm{T}$ he quality of fundus images including angiographic mages may be suboptimal for many reasons, such as defocus, media opacities, and small pupils. It is often important to have the best available images particular for the diagnosis and treatment of retinal diseases including choroidal neovascularisation (CNV) and other problems. High speed angiography ${ }^{1-4}$ has been extensively used to characterise the appearance of the retinal and choroidal circulation using fluorescein or indocyanine green dye.

One of the difficulties with using high speed angiography is the short exposure time for each image. Nevertheless, this is not usually a problem in the early or mid-phase of the angiogram, since sufficient fluorescence is present in this phase. However, during late phase angiography the short exposure time can severely limit the brightness of the angiogram. Therefore the signal to noise ratio (SNR) can be very low at these low light levels. Hence, this condition results in a dramatic reduction in image quality for clinical diagnosis.

Because of the American National Standard Institute (ANSI) safety standards, ${ }^{5}$ the only method to improve SNR is to reduce noise in the image. One of the standard technique of reducing image noise is to record a rapid series of images and average the signal over the images in the series. ${ }^{6-15}$ Swindell and Mosleh-Shirazi have analysed the theoretical improvement that can be achieved by averaging serial images to reduce $\mathrm{SNR}^{13}$ and found that the improvement is proportional to the square root of the number of images. However, in ophthalmic imaging applications eye motions limit the number of images that can be included. Alternatively, George and colleagues have used a digital "sharpen" filter to enhance grading of diabetic retinopathy photographs. $^{16}$

The goal of this study was to determine whether image averaging techniques using near video rate rapid sequence images taken with a confocal scanning video laser ophthalmoscope (SLO), resulted in improvement of image quality in eyes with CNV and age related macular degeneration (AMD). The images of most interest in terms of improving image quality were images of normally poor quality and large amount of noise such as late phase fluorescein angiography (FA) and indocyanine green angiography (ICGA) images and images taken during oral FA.

\section{METHODS}

Over a 3 month period, 27 eyes of 20 patients underwent angiography using intravenous (IV) FA, IV ICGA, or oral FA or a combination of several angiography methods. A total of 30 image sequences were recorded. Ten of these patients underwent IV ICGA, 10 underwent IV FA, and 10 oral FA. The cases were sequential cases and were not preselected. The patients were examined in the Jacobs Retina Center at the University of California San Diego (UCSD) Shiley Eye Center, Department of Ophthalmology. The Shiley Eye Center is tertiary referral clinic for diseases of the retina. The human research protection program at UCSD has reviewed and approved this study.

We have used a confocal SLO (Heidelberg retina angiograph, HRA, Heidelberg Engineering Inc, Vista, CA, USA) to record FA or ICGA as well as infrared reflectance images. The instrument has been described previously ${ }^{3}{ }^{4}$ and has four different laser wavelengths for illumination of the fundus $(488 \mathrm{~nm}$ for FA excitation, $514 \mathrm{~nm}$ for red-free imaging, $795 \mathrm{~nm}$ for ICGA excitation, and $840 \mathrm{~nm}$ for infrared imaging). The HRA scans a laser point across the retina with the approximate size of $15 \times 15 \mu \mathrm{m}$, the approximate lateral resolution of the eye. ${ }^{17-19}$ Since a one dimensional light detector is used, the inherent random noise is spread evenly

Abbreviations: $A M D$, age related macular degeneration; CNV, choroidal neovascularisation; FA, fluorescein angiography; FOV, field of view; HRA, Heidelberg retina angiograph; ICGA, indocyanine green angiography; IV, intravenous; RPE, retinal pigment epithelium; SLO, scanning laser ophthalmoscope; SNR, signal to noise ratio 


\begin{tabular}{ll} 
Table 1 Study population & \\
\hline Diagnosis & Number of eyes \\
\hline Active choroidal neovascularisation & 14 \\
associated with AMD & 3 \\
Disciform scar or old inactive CNV & 8 \\
Dry AMD with drusen & 2 \\
Macular pathology & \\
\hline
\end{tabular}

$A M D$, age related macular degeneration; $C N V$, choroidal neovascularisation.

across the entire image. Software was used to average multiple images from a 10-20 image series (over 0.81.7 seconds) (Heyex software 1.3.2.0 with HRA viewer module 2.1.0.0, Heidelberg Engineering, Heidelberg, Germany).

For intravenous FA or ICGA, images were acquired electronically using the HRA. The alignment and averaging functions are part of the Heidelberg Eye Explorer software. A digital angiographic sequence was acquired in the early, mid, and or late phase of the angiogram. All angiograms were taken with only one dye at a time at an image acquisition rate of 12 images per second $(512 \times 512$ pixel $)$ at $30^{\circ} \times 30^{\circ}$ field of view (FOV). The digital angiographic sequences were later processed by the HRA software to derive an image averaged image with the help of an adaptive algorithm (Tilman Otto, personal communication). This algorithm employs motion compensated image averaging to improve picture quality. In the first step a cross correlation algorithm is used to align individual images of the video sequence with respect to each other. In the second step the aligned image sequence is averaged to yield the resultant image. Since the noise is randomly distributed, the image averaged image has an improved image quality. We did not record simultaneous FA/ ICGA images since these images have only $256 \times 256$ pixel resolution per image. In one case we recorded a $10^{\circ} \times 10^{\circ} \mathrm{FOV}$ for illustration.

Image quality was assessed by two different methods. The first method used two masked observers (MEB and AEM) who graded the image improvement on a scale of $0-3$. Individual (non-averaged) images were compared with averaged images taken from the same time during the study. Grade 0 means that both images were of equal quality. For each image pair we determined if one image was mildly (grade 1), moderately (grade 2 ), or highly superior (grade 3 ) to the other. A positive score was recorded in the database after unmasking if the image averaged image was judged to be of higher quality than the non-averaged image, otherwise a negative score was recorded. The mean improvement of
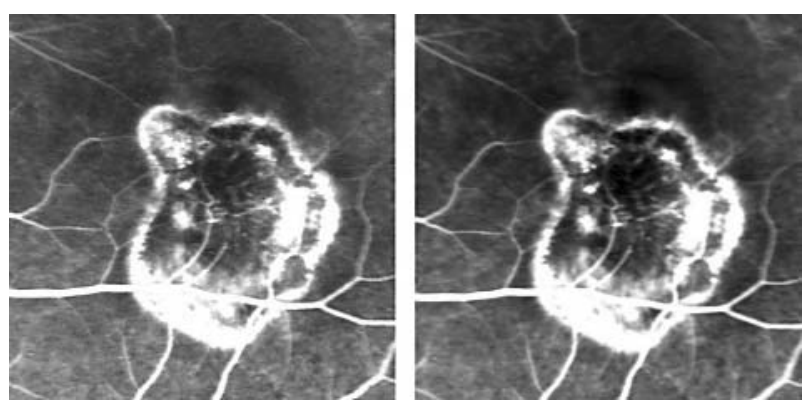

Figure 1 Intravenous fluorescein angiogram of a 31 year old female patient with myopic choroidal neovascularisation at high resolution $\left(10^{\circ} \times 10^{\circ}\right.$ field of view $)$. Mid phase angiogram is seen with the averaged image on the right. The retinal vascular landmarks at the edge of the leaking membrane are seen in more detail on the averaged image than the raw image (left).
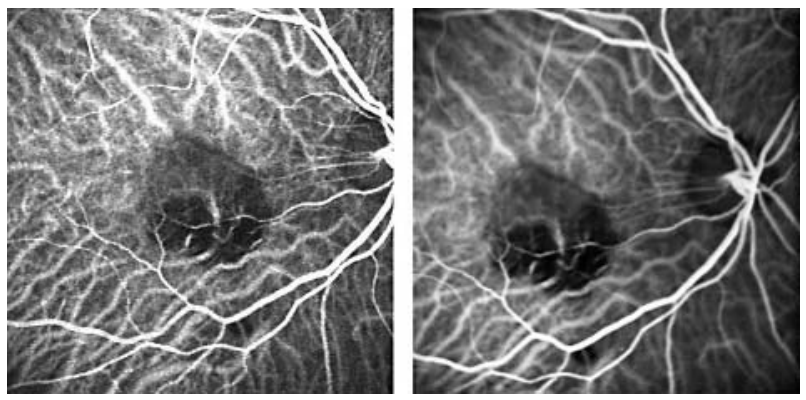

Figure 2 An early phase ICG angiogram showing neovascularisation with its feeder vessel in the right eye of a 73 year old female. The raw image (left) shows a moderate amount of noise. The averaged image (right) shows more detail of the branches of the feeder vessel, but the improvement in quality is mild. This is a typical example of our experience with early phase ICG angiography.

each phase and type of angiogram and statistical significance by one sample $t$ test was determined.

The second, more quantifiable, method was performed on an imaging workstation (Macintosh Powerbook G4, Apple, Cupertino, CA, USA) with Adobe Photoshop (Photoshop 5.5, Adobe Systems Inc, San Jose, CA, USA). One of the authors (DUB) was masked to the type and phase of the angiogram. For each of the 30 image pairs the operator added Gaussian noise to the image with apparent better visual quality. This would allow quantification of the amount of image improvement by degrading the image averaged, improved image back to the level of the original image. The image processing software allows adding a reproducible, variable amount of noise ranging from 1 to 999 in arbitrary units. The amount of added noise was recorded and noted as the amount of image improvement.

\section{RESULTS}

Thirty image sequences were recorded in 27 eyes of 20 sequential patients with age related macular degeneration over a 3 month period (table 1). Of these 27 eyes, 14 had active CNV associated with AMD. Three eyes had disciform scars or old inactive choroidal neovascularisation. Eight eyes had dry AMD with drusen and were examined by angiography to rule out CNV. An additional two eyes had macular pathology associated with AMD.

The results of the subjective grading by two ophthalmologists are shown in table 2 . There was significant improvement in all groups and all phases. In the early phase (fig 1) of the IVFA five out of 10 subjects showed significant improvement $(p<0.05)$ while seven out 10 subjects showed significant improvement in the IVFA mid-phase $(p<0.01)$. In the early phase of the ICGA (fig 2) eight out of 10 subjects showed significant improvement $(\mathrm{p}<0.01)$ while nine out 10 subjects showed significant improvement in the ICGA midphase $(\mathrm{p}<0.001)$. The improvement was also highly significant $(\mathrm{p}<0.001)$ in the late phase of FA (fig 3$)$, the mid and late phase of ICGA, and all phases of the oral FA (midphase: fig 4, late phase: figs 5-7). It has to be noted that the appearance of the choroidal circulation was particularly improved (figs 2-7). The speckle-like noise that is present in the original images was largely absent in the averaged images.

The accuracy of the motion correcting image alignment process can easily be evaluated by the appearance of the major blood vessels in the aligned image. If the alignment was successful, no double blood vessels appeared around the optic nerve head. Unsuccessful correction of lateral or rotational motion was characterised by the double 

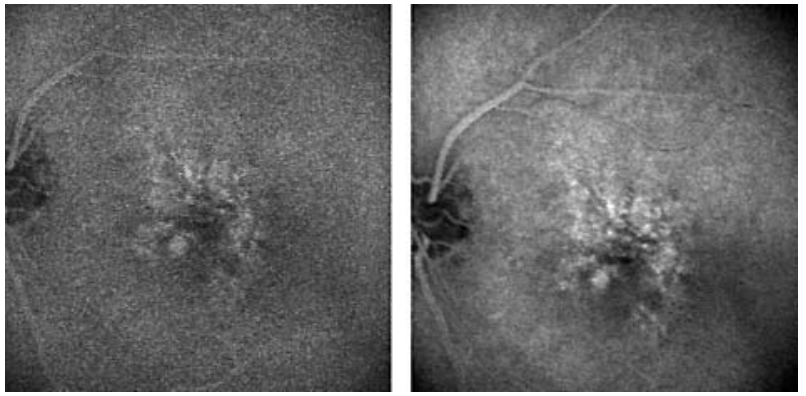

Figure 3 This is a 30 minute late oral fluorescein angiogram in the left eye of a 74 year old male patient with atrophic macular degeneration. The raw image is shown on the left. The second and third order retinal vessels are seen in better detail in the averaged image (right) as are the detail of the staining drusen and retinal pigment epithelium (RPE) window defects.
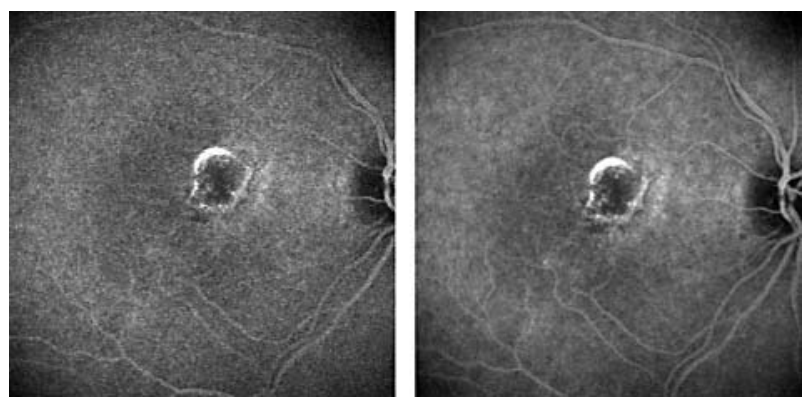

Figure 4 Right eye of 71 year old female patient with macular degeneration status after focal laser treatment developed a recurrence at the margin. This mid-phase oral FA shows leakage in both the averaged (right) as well as the raw image (left). The retinal vascular landmarks are better seen in the averaged image.

appearance of one or more blood vessels. We did not observe any unsuccessfully aligned image series in this study.

The results of our more quantifiable image evaluation technique are shown in table 3 . The numbers indicate the amount of noise that was added to the image averaged image to make it appear equal to the raw noisy image. Thus, a higher number indicates that the image averaged image had less noise and was thus of higher quality. The results show that the more quantifiable image evaluation and comparison method follows the trend of the subjective grading results shown in table 2 . The images with the highest improvement were recorded in the late phase of the FA, late phase of the ICGA, and all phases of oral FA.
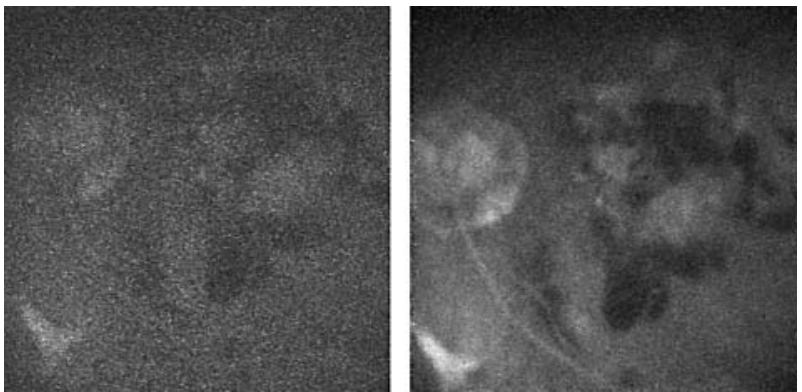

Figure 5 Late stage intravenous fluorescein angiogram in left eye image of a 92 year old female with dense cataract. The averaged image (right) showed detail of CNV and surrounding haemorrhage much better than seen in the raw image (left).
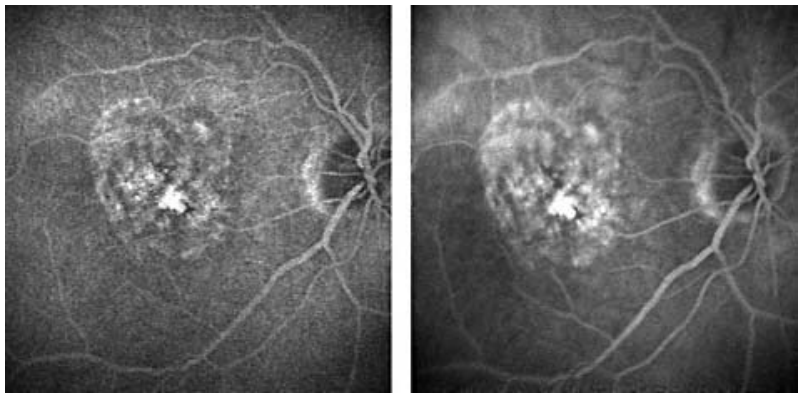

Figure 6 Late stage oral fluorescein angiogram in the right eye of an 84 year old female patient with small choroidal neovascular membrane in an area of RPE atrophy. The averaged image on the right (late phase) shows improved detail of retinal vascular landmarks surrounding the lesions.

\section{DISCUSSION}

CNV associated with AMD is treatable but often with poor results. ${ }^{2021}$ Feeder vessel treatment has been evaluated ${ }^{22-24}$; however, such vessels often are difficult to discern. Improved visualisation of CNV might be of benefit in allowing improved classification of the disease, and earlier or better treatment. For this reason we wanted to determine whether image averaging would improve visualisation of $\mathrm{CNV}$ in patients with AMD. In addition, oral $\mathrm{FA}^{25}$ has recently been shown to provide clinically useful images in many eyes and we wished to determine if image quality could be improved. Even in eyes without CNV but with pre-neovascular lesions it can be very important to rule out CNV and poor image quality can make this difficult.

In our study we found that image averaging significantly improves image quality in a subjective, four level grading examination and a more quantifiable, Gaussian noise additive grading examination. The improvement is real, albeit modest in the early and mid-phase of the angiograms. The improvement may be clinically useful.

Image averaging reduces noise, but loses some temporal information. Rapid changes occur only during the arterial inflow phase and we did not attempt image averaging in this phase. Furthermore, as every ophthalmologist is aware, the image quality in the inflow phase is usually sufficient and would not benefit from image quality improvement.

Image averaging is a standard technique used in all areas of medical imaging for improving images. ${ }^{6-15} \mathrm{~A}$ theoretical analysis shows that the improvement of the SNR in the averaged image increases with the square root of the number of images. ${ }^{13}$ This was confirmed in a three dimensional reconstruction of an electron microscopy tomography study ${ }^{14}$
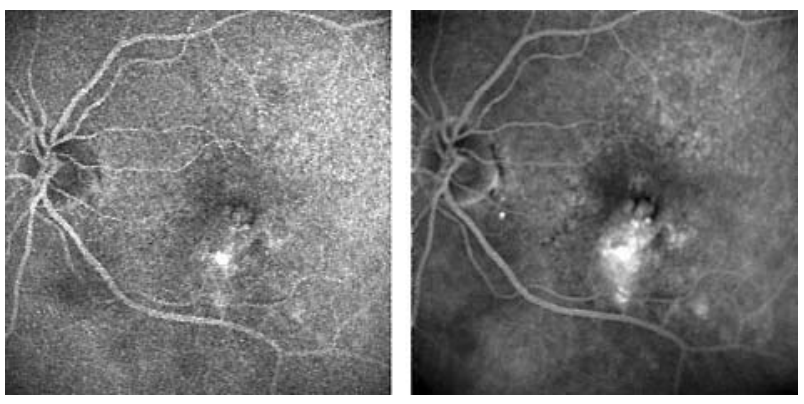

Figure 7 An 87 year old female patient with an ill defined choroidal neovascular membrane seen on a late phase of an oral fluorescein angiogram. The averaged image (right) showed improved detail of leaking area and overlying retinal areas compared to the raw image (left). 
Table 2 Magnitude of improvement in subjective image quality assessment (SD)

\begin{tabular}{lllll}
\hline $\begin{array}{l}\text { Angiogram } \\
\text { type }\end{array}$ & $\begin{array}{l}\text { Number } \\
\text { of images }\end{array}$ & Early phase & Mid-phase & Late phase \\
\hline IV FA & 10 & $0.80(0.92)^{*}$ & $1.00(0.82)^{* *}$ & $2.10(0.99)^{* * \star}$ \\
ICGA & 10 & $1.20(0.79)^{* *}$ & $1.80(0.92)^{* \star *}$ & $2.30(0.48)^{* * *}$ \\
Oral FA & 10 & $1.56(0.88)^{* * *}$ & $2.22(0.83)^{* * *}$ & $2.44(0.53)^{* * *}$ \\
\hline
\end{tabular}

Scale: Grade 0 means that both images were of equal quality. For each image pair we determined if one image was mildly (grade 1), moderately (grade 2), or highly superior (grade 3) * $p<0.05$ in one paired Student $t$ test comparing to null hypothesis. " $\mathrm{p}<0.01$ in one paired Student $t$ test comparing to null hypothesis. ${ }^{* * *} p<0.001$ in one paired Student $t$ test comparing to null hypothesis.

Table 3 Magnitude of improvement in quantitative image quality assessment (SD)

\begin{tabular}{llll}
\hline $\begin{array}{l}\text { Angiogram } \\
\text { type }\end{array}$ & Early phase & Mid-phase & Late phase \\
\hline IV FA & $15(8.9)$ & $17.5(13.4)$ & $21.5(13.4)$ \\
ICGA & $15.3(4.5)$ & $15.5(6.4)$ & $18.8(10.3)$ \\
Oral FA & $19.8(9)$ & $16.1(7.8)$ & $22.7(6)$ \\
\hline
\end{tabular}

Scale: The improvement is given in arbitrary units. Adobe Photoshop version 5.5 (Macintosh) was used to add Gaussian noise. The variable amount of noise that can be added ranges from 0 to 999 units.

and in magnetic resonance imaging studies. ${ }^{15}$ Some authors have reported problems with the technique in the presence of motion artefacts. ${ }^{7}$ The main problem is that uncompensated motion artefacts degrade the image rather than allow image improvement. Our experience was similar with non-aligned or poorly aligned image series. However, the presence of the automated cross correlation image alignment enables us to align movement. ${ }^{26}$ The inability of the software to align some images can easily be spotted by the appearance of double images in the blood vessel structure.

The main problem in the acquisition of angiographic images is the low fluorescence in the late phase of both FA and ICGA. Other problems are the poor resolution caused by the aberrations in the eye and the light safety limits. Thus, improved optics, or improved detectors, or improved dyes can improve visibility. Adaptive optics ${ }^{27-31}$ has not only the potential to improve resolution, but should also improve the SNR as more light from the retina is collected at the light detector. Since light levels in adaptive optical imaging are limited by the same rules as in our present study, image averaging is a useful and helpful method that should be employed as a routine imaging method in digital retinal imaging. Future improvements to the image averaging technique include improved automated cross correlation with detection and automatic rejection of unaligned images and inclusion of eye trackers. ${ }^{32}$

Image averaging improved image quality for all phases of oral and intravenous fluorescein angiography and intravenous ICG angiography. Improvement in image quality is most pronounced in the late phase of the angiograms. The improvement also depends on the type of angiography that was studied. The improvement was least in intravenous FA and best in oral FA. For ICG angiography there was also striking improvement in late phase image quality.

We believe that the degree of improvement depends on the initial quality of the acquired image. IV FA has an inherent high quality in the early phase. In this case image averaging can only produce a marginal improvement. Oral FA and ICGA are subject to more noise influence that can be ameliorated with noise reducing techniques such as image averaging. Our group and others have recently used oral $\mathrm{FA}^{25}{ }^{33}$ for imaging patients with AMD and diabetic retinopathy. Newsom and associates have used "local contrast enhancement" to improve image quality in oral FA. ${ }^{34}$ Thus, new types of imaging methods in ophthalmology require new image processing tools. In theory, the use of image averaging is only limited by image registration problems and the increase in dynamic range as a result of the additive nature of the averaging process.

In conclusion, image averaging offers a simple yet effective method to significantly improve image qualify for clinical diagnosis in angiography. This technique merits further investigation.

\section{Authors' affiliations}

D U Bartsch, W R Freeman, Jacobs Retina Center, Department of Ophthalmology, University of California San Diego, CA, USA M H El-Bradey, Faculty of Medicine, Tanta University, Egypt A El-Musharaf, Toronto, Canada

Sponsor details/grant support: This study was sponsored in part by a grant from the National Eye Institute NIH-NEI grant \#EY13304 (DUB) and NIH grant \#EY07366 (WRF) and Research to Prevent Blindness (UCSD).

Competing interests: The UCSD Shiley Eye Center has received unrestricted research support from Heidelberg Engineering, a company that makes the confocal scanning laser ophthalmoscope used in this study.

Ethics approval: The Human Research Protection Program at UCSD has reviewed and approved this study.

\section{REFERENCES}

1 Scheider A, Schroedel C. High resolution Indocyanine green angiography with a scanning laser ophthalmoscope. Am J Ophthalmol 1989;108:458-9.

2 Flower RW. Extraction of choriocapillaris hemodynamic data from ICG fluorescence angiograms. Invest Ophthalmol Vis Sci 1993;34:2720-9.

3 Bartsch DU, Weinreb RN, Zinser G, et al. Confocal scanning infrared laser ophthalmoscopy for indocyanine green angiography. Am J Ophthalmol 1995; 120:642-51.

4 Freeman WR, Bartsch DU, Mueller AJ, et al. Simultaneous indocyanine green and fluorescein angiography using a confocal scanning laser ophthalmoscope [see comments]. Arch Ophthalmol 1998;116:455-63.

5 ANSI Z136.1. American national standard for the safe use of lasers. 3rd ed. New York: American National Standards Institute, 1993: 1-124.

6 Kavanaugh KM, Pinto IM, McGillem MJ, et al. Effects of video frame averaging, smoothing and edge enhancement on the accuracy and precision of quantitative coronary arteriography. Int J Card Imaging 1990;5:233-9.

7 Leszczynski KW, Shalev S, Cosby NS. An adaptive technique for digital noise suppression in on-line portal imaging. Phys Med Biol 1990;35:429-39.

8 Yee T. Image processing software for enhanced visualization of faint or noisy autoradiographic images. Biotechniques 1991;10:786-9.

9 Rajapakshe R, Shalev S. Noise analysis in real-time portal imaging. I. Quantization noise. Med Phys 1994;21:1263-8. 
10 Woodward JT, Kono C, Madsen LL, et al. Inherent bias in correlation averaged images. J Microsc 1995; 178:86-92.

11 Madore B, Henkelman RM. A new way of averaging with applications to MRI. Med Phys 1996;23:109-13.

12 Török B, Niederberger $H$, Bischoff P. [Simultaneous digital indocyanine green and fluorescein angiography]. Klin Monatsbl Augenheilkd 1996;208:333-6.

13 Swindell W, Mosleh-Shirazi MA. Noise reduction by frame averaging: a numerical simulation for portal imaging systems. Medical Physics 1995;22:1405-11.

14 Ofverstedt LG, Zhang K, Isaksson LA, et al. Automated correlation and averaging of three-dimensional reconstructions obtained by electron tomography. J Struct Biol 1997; 120:329-42.

15 Holmes CJ, Hoge R, Collins L, et al. Enhancement of MR images using registration for signal averaging. J Comput Assist Tomogr 1998;22:324-33.

16 George LD, Lusty J, Owens DR, et al. Effect of software manipulation (Photoshop) of digitised retinal images on the grading of diabetic retinopathy. Br J Ophthalmol 1999;83:911-3.

17 Bartsch DU, Freeman WR. Laser-tissue interaction and artifacts in confocal scanning laser ophthalmoscopy and tomography. Neurosci Biobehav Rev 1993;17:459-67.

18 Bartsch DU, Freeman WR. Axial intensity distribution analysis of the human retina with a confocal scanning laser tomograph. Exp Eye Res 1994:58:161-73.

19 Weinreb RN, Lusky M, Bartsch DU, et al. Effect of repetitive imaging on topographic measurements of the optic nerve head. Arch Ophthalmol 1993; 111:636-8.

20 Freund KB, Yannuzzi LA, Sorenson JA. Age-related macular degeneration and choroidal neovascularization. Am J Ophthalmol 1993;115:786-91.

21 Gelfand YA, Linn S, Miller B. The application of the macular photocoagulation study eligibility criteria for laser treatment in age-related macular degeneration. Ophthalmic Surg Lasers 1997;28:823-7.

22 Lee WK, Kim HK. Feeder vessel laser photocoagulation of subfoveal choroidal neovascularization. Korean J Ophthalmol 2000;14:60-8.

23 Shiraga F, Ojima Y, Matsuo T, et al. Feeder vessel photocoagulation of subfoveal choroidal neovascularization secondary to age-related macular degeneration. Ophthalmology 1998;105:662-9.
24 Staurenghi G, Orzalesi N, La Capria A, et al. Laser treatment of feeder vessels in subfoveal choroidal neovascular membranes: a revisitation using dynamic indocyanine green angiography. Ophthalmology 1998; 105:2297-305.

25 Garcia CR, Rivero ME, Bartsch DU, et al. Oral fluorescein angiography with the confocal scanning laser ophthalmoscope. Ophthalmology 1999;106:1114-18.

26 Rivero ME, Bartsch DU, Otto $T$, et al. Automated scanning laser ophthalmoscope image montages of retinal diseases. Ophthalmology 1999; 106:2296-300.

27 Liang J, Williams DR. Adaptive optics for high-resolution imaging of the living human retina. CLEO '96. Summaries of Papers Presented at the Conference on Lasers and Electro-Optics. Vol.9. 1996 Technical Digest Series. Conference Edition (IEEE Cat No 96CH35899), 1996:74.

28 Marcos S, Navarro R, Artal P. Coherent imaging of the cone mosaic in the living human eye. J Optical Soc Am A (Optics, Image Science and Vision) 1996;13:897-905.

29 Zhu LJ, Bartsch DU, Freeman WR, et al. Modeling human eye aberrations and their compensation for high-resolution retinal imaging. Optom Vis Sci 1998;75:827-39

30 Zhu L, Sun PC, Bartsch DU, et al. Adaptive control of a micromachined continuous-membrane deformable mirror for aberration compensation. Applied Optics 1999;38:168-76.

31 Zhu L, Sun PC, Bartsch DU, et al. Wave-front generation of Zernike polynomial modes with a micromachined membrane deformable mirror. Applied Optics 1999;38:6019-26.

32 Ferguson RD, Hammer DX, Elsner AE, et al. Wide-field retinal hemodynamic imaging with the tracking scanning laser ophthalmoscope. Optics Express 2004; 12:5198-208

33 Newsom R, Moate B, Casswell T. Screening for diabetic retinopathy using digital colour photography and oral fluorescein angiography. Eye 2000; 14:579-82.

34 Newsom RS, Sinthanayothin C, Boyce J, et al. Clinical evaluation of 'local contrast enhancement' for oral fluorescein angiograms. Eye 2000;14:318-23. 